\title{
Colloidal brazil nut effect in sediments of binary charged suspensions
}

\author{
Ansgar Esztermann, Hartmut Löwen \\ Institut für Theoretische Physik II, Heinrich-Heine-Universität Düsseldorf, \\ Universitätsstraße 1, D-40225 Düsseldorf, Germany
}

(Dated: January 2nd, 2004)

\begin{abstract}
Equilibrium sedimentation density profiles of charged binary colloidal suspensions are calculated by computer simulations and density functional theory. For deionized samples, we predict a colloidal "brazil nut" effect: heavy colloidal particles sediment on top of the lighter ones provided that their mass per charge is smaller than that of the lighter ones. This effect is verifiable in settling experiments.

PACS numbers: 82.70.Dd, 61.20.Ja, 05.20.Jj
\end{abstract}

Binary systems of granular matter separate upon shaking in gravity, so that the larger particles lie on top of the smaller ones even if they are heavier and denser than the latter. This is due to a sifting mechanism in which tiny grains filter through the interstices between the large particles which is well-known as "brazil nut" effect: in a jar of mixed nuts or in a package of cereal, the largest species rises to the top 1, 2]. This clearly distinguishes granular matter from ordinary fluids where the rising species is controlled by Archimedes' law. Understanding the full details of the brazil nut effect is still a problem; recently even a reverse brazil nut effect of large light grains sinking in a granular bed has been predicted [3, 4, 5] and verified in experiments [6].

In this letter, we report on equilibrium density profiles of binary charged colloidal fluids ("macroions") under gravity. Using extensive Monte-Carlo computer simulations of the "primitive" model [7] of strongly asymmetric electrolytes and a density functional theory, we predict that the heavier particles sediment on top of the lighter ones provided the charge per mass of the heavier particles is higher. In analogy to granular matter, we call this counter-intuitive phenomenon a colloidal brazil nut effect. It is generated by the entropy of the microscopic counterions in the solution, which are coupled to the macroions by strong Coulomb binding. Clearly, though this effect is qualitatively similar to the granular brazil nut effect insofar as heavy particles are on top of lighter ones, its physical origin is different: first, the particle charge (and not the size) is crucial. Second, the colloidal brazil nut effect is a pure equilibrium phenomenon while the granular brazil nut effect happens intrinsically in non-equilibrium. The colloidal brazil nut effect can be verified, e.g., in depolarized-light scattering or real-space experiments on sediments of strongly deionized binary charged suspensions [8, 9]. Similar techniques have been used to measure one-component colloidal density profiles 9, 10 where deviations from the ideal barometric law 11] are still an ongoing debate [12, 13, 14, 15, 16].

We simulate the asymmetric "primitive" model of binary charged suspensions in which the solvent only enters via a continuous dielectric background with permittivity $\epsilon$ but all charged particles (two species of negatively charged macroions and microscopic counter- and coions) are treated explicitly at constant temperature $T$. With $Z_{1} e, Z_{2} e,-q e$ and $\sigma_{1}, \sigma_{2}, \sigma_{c}$ denoting the charges and the diameters of the two colloidal species and the microions, the interaction between the charged particles is given as a combination of Coulomb forces and excluded volume of the hard particle cores. Here, $e$ is the electron charge. For simplicity we assume that the co- and counterions of the salt solution have the same valency and the same hard core diameter $\sigma_{c}$. We consider a finite system of $N_{1}, N_{2}$ charged macroions and corresponding number of counterions (fixed by global charge neutrality) plus salt ions of bulk concentration $c_{s}$. The simulation box is rectangular with lengths $L_{x}=L_{y}$ and $L_{z}=32 L_{x}$ in the three different spatial directions with periodic boundary conditions in $x$ and $y$ direction and finite length in $z$ direction. Hard walls are placed at $z=0, L_{z}$ and gravity with acceleration $g$ points along the $-z$ direction. Only the colloidal particles with their buoyant masses $m_{1}$ and $m_{2}$ are subject to gravity, whereas the microions are not.

A Monte-Carlo simulation is performed in the canonical ensemble with the long-ranged Coulomb interaction treated via Lekner sums [17]. Typically $10^{3}$ Monte Carlo moves per particle were performed for equilibration and it took an additional $10^{4}$ Monte Carlo moves per particle to gather statistics. Finite system size effects were carefully checked by changing all lateral linear dimensions by a factor of 4 . This means that we have changed the total number of colloidal particles $N_{1}+N_{2}$ in the range of $12-$ 200. We have calculated the inhomogeneous $z$-dependent averaged density profiles $\rho_{1}(z), \rho_{2}(z), \rho_{3}(z)$ and $\rho_{4}(z)$ of the two macroions and the counter- and coions. Data are shown for the largest system size where $N_{1}=N_{2}=100$.

Besides the ratios $Z_{1} / q, Z_{2} / q, m_{1} / m_{2}, \sigma_{1} / \sigma_{2}, \sigma_{c} / \sigma_{2}$, the system is characterized by two partial area densities $n_{i}=N_{i} / L_{x} L_{y}(i=1,2)$, the Bjerrum length $\lambda_{B}=q^{2} e^{2} / \epsilon k T$ ( $k T$ denoting the thermal energy), the gravitational length $\ell_{2}=k T / m_{2} g$ of the second particle species, and the salt concentration. In order to reduce the parameter space, we have assumed throughout the simulations monovalent microions $(q=1)$, and the same hard core diameter of the macroions $\sigma=\sigma_{1}=\sigma_{2}$ which serves as a natural length scale. We further fixed $Z_{2}=15, \lambda_{B}=\sigma_{c}=\sigma / 128$ and $n_{1}=n_{2}=0.1 / \sigma^{2}$. This corresponds to typical parameters for low-charge aque- 


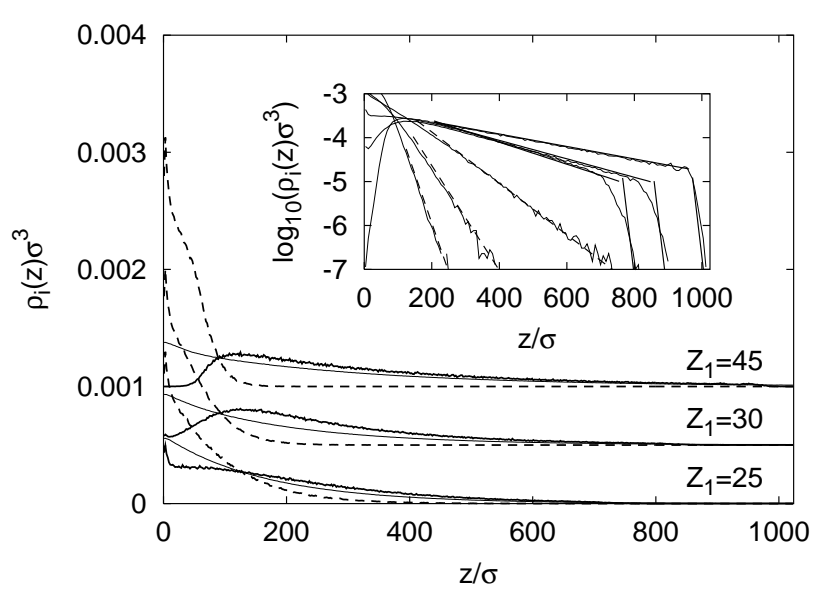

FIG. 1: Density profiles of macro- and counterions for $Z_{1}=$ 45, 30, 25 (solid lines; top to bottom). The second colloidal component is shown as a dashed line. Counterion densities (thin lines) have been divided by $Z_{1}+Z_{2}$. For clarity, curves pertaining to different simulation runs have been shifted by $5 \times 10^{-4}$ with respect to each other. The parameters are: $m_{1} / m_{2}=1.5, \ell_{2} / \sigma=10, c_{s}=0$. Inset: Semi-logarithmic plots of the same colloidal density profiles with the slopes predicted from theory.

ous suspensions. We varied the colloidal charge $Z_{1}$, the colloidal mass ratio $m_{1} / m_{2}$ (with $m_{1}>m_{2}$ ), the gravitational length $\ell_{2}$, and the salt concentration.

In the salt-free case, density profiles for the two macroions and the counterions are shown in Figure 1 for $m_{1} / m_{2}=1.5$ and three different macroion charges $Z_{1}=45,30,25$. For large heights $z$, the heavy particles (solid curve) are on top of the lighter ones (dashed curve). This colloidal brazil nut effect is getting stronger for increasing charge asymmetry between the colloidal particles: for the highest charge $Z_{1}=45$, the two macroion species are almost completely separated. To quantify the brazil nut effect, we define - in analogy to the ordinary brazil nut problem [5] - a mean (or sedimentation) height of the two profiles via

$$
h_{i}=\frac{\int_{0}^{\infty} z \rho_{i}(z) d z}{\int_{0}^{\infty} \rho_{i}(z) d z} \quad i=1,2
$$

By definition, the colloidal brazil nut effect occurs if $h_{1}>$ $h_{2}$, but there is no brazil nut effect (or a "reverse" brazil nut effect) in the opposite case, $h_{1} \leq h_{2}$.

Let us now describe the properties of the density profiles for increasing $z$ qualitatively and put forward a simple theory to describe the basic features. Close to the hard container bottom at $z=0$, small correlation effects are visible as a density shoulder of the low-charge particles while high charge particles are depleted from the wall. The reason is a combination of a pure interface effect and Archimedes' principle. First, we have checked by simulation that depletion of high-charge particle persists for zero gravity. Second, by crudely mapping the interacting binary colloidal mixture onto one with effec- tive hard interaction cores, the high-charge particles will have a larger diameter and hence Archimedes' principle will lift the high-charge particles in the sea of small ones provided their mass density is smaller [18]. For our parameters, however, the latter effect is small and confined to regions close to the wall. More importantly, as revealed by the semi-logarithmic plot in the inset of Figure 1 there is an exponential decay of the two colloidal profiles for intermediate heights associated with two different decay lengths for the two colloidal species. The decay length for the heavier but high-charge particles is larger than that for the low-charge particles. This gives the most significant contribution in the integral (1) of the mean height. Finally, for large $z$, there is cross-over towards another exponential decay involving the gravitational length $\ell_{1}=k T / m_{1} g$ for the high-charge particles. It is important to note that the density profiles fulfill local charge neutrality throughout the whole sample except at the container bottom $(z=0)$ and at very large heights $z$. But even there the excess charge separated is small [19].

Our theoretical explanation for the colloidal brazil nut effect is based on a simple density functional approach. The free energy $\mathcal{F}$ per unit area, which is a functional of the inhomogeneous density fields $\rho_{1}(z), \rho_{2}(z), \rho_{3}(z)$ of the macro- and counterions, splits into the gravitational energy, the entropy of the three species and all Coulomb contributions. The latter are approximated within a mean-field-type Poisson-Boltzmann theory [12, 13, 14, 15, 16]. Hence:

$$
\begin{aligned}
\mathcal{F} & {\left[\rho_{1}(z), \rho_{2}(z), \rho_{3}(z)\right]=\sum_{\nu=1}^{2} \int_{0}^{\infty} d z m_{\nu} g z \rho_{\nu}(z) } \\
& +\sum_{\nu=1}^{3} \int_{0}^{\infty} d z k T \rho_{\nu}(z)\left(\ln \left(\Lambda_{\nu}^{3} \rho_{\nu}(z)\right)-1\right) \\
& +\frac{1}{2} \int d^{2} r^{\prime} \int_{0}^{\infty} d z \int_{0}^{\infty} d z^{\prime} \frac{\rho_{t}(z) \rho_{t}\left(z^{\prime}\right)}{\epsilon \sqrt{r^{\prime 2}+\left(z-z^{\prime}\right)^{2}}}
\end{aligned}
$$

Here, $\Lambda_{\nu},(\nu=1,2,3)$, are Lagrange multipliers which ensure that the overall colloidal densities per unit area equal the prescribed number densities, i.e. $\int_{0}^{\infty} d z \rho_{i}(z)=$ $n_{i}(i=1,2) . \quad \Lambda_{3}$ is fixed by global charge neutrality, and $\rho_{t}(z)=Z_{1} e \rho_{1}(z)+Z_{2} e \rho_{2}(z)-q e \rho_{3}(z)$ is the total local charge density of the system. The functional $\mathcal{F}$ is minimal for the physically realized equilibrium density profiles.

We discuss two different cases of weak and strong Coulomb coupling subsequently. For weak Coulomb coupling, which is realized for very large heights $z$ where the densities are extremely small, one may neglect the third term on the right hand side of Eq. (2). Then, the minimization of $\mathcal{F}$ yields colloidal density profiles which follow the traditional barometric law $\rho_{i}(z) \propto \exp \left(-z / \ell_{i}\right)$ $(i=1,2)$. Strong Coulomb coupling, on the other hand, will impose local charge neutrality $\rho_{t}(z)=0$ which implies that the counterion density field is enslaved to that of the macroions. Minimizing $\mathcal{F}$ in this limit with respect 


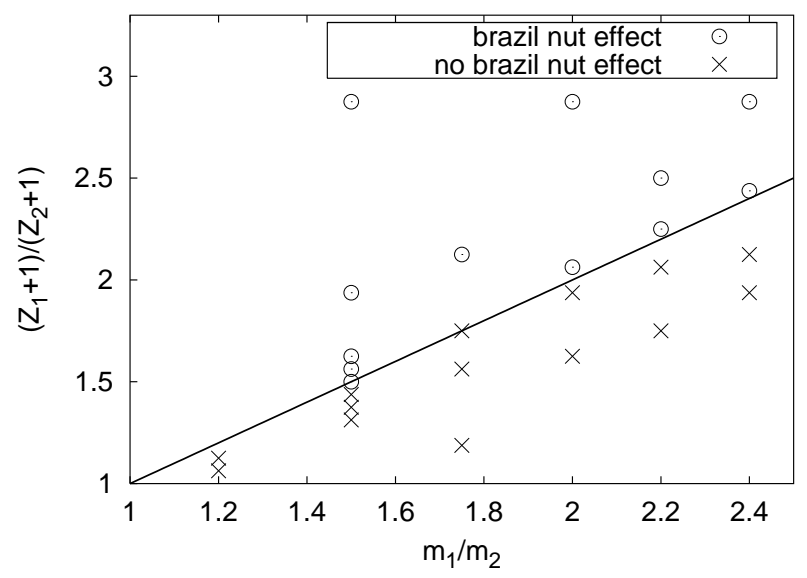

FIG. 2: Crossover towards the brazil nut effect. Shown are different charge asymmetries $\left(Z_{1}+1\right) /\left(Z_{2}+1\right)$ and mass ratios $m_{1} / m_{2}$ for which the brazil nut effect was (circles) or was not observed (crosses). The straight line shows the theoretical prediction. The parameter combinations were obtained by changing $\ell_{2}$ and $Z_{1}$ is the range of $4 \ldots 20 \sigma$ and $16 \ldots 45$, respectively.

to the two colloidal densities only, again yields an exponential decay $\rho_{i}(z) \propto \exp \left(-\gamma_{i} z\right)(i=1,2)$ but with inverse decay lengths $\gamma_{i}$ which are smaller than $1 / \ell_{i}$. These decay lengths $\gamma_{i}$ turn out to be as follows: Let $\alpha$ be the index $i$ for which the mass per charge ratio $\frac{m_{i}}{Z_{i} / q+1}$ is minimal and let $\beta$ be the index complementary to $\alpha$, i.e. $\beta=1$ if $\alpha=2$ and $\beta=2$ if $\alpha=1$. Then

$$
\gamma_{\alpha}=\frac{m_{\alpha}}{Z_{\alpha} / q+1} \frac{g}{k T}
$$

and

$$
\gamma_{\beta}=\left(m_{\beta}-m_{\alpha} \frac{Z_{\beta} / q}{Z_{\alpha} / q+1}\right) \frac{g}{k T}>\gamma_{\alpha}
$$

with $\gamma_{\beta} \geq \frac{m_{\beta} g}{k T\left(Z_{\beta} / q+1\right)} \geq \gamma_{\alpha}$. This second case of strong Coulomb coupling will be realized for heights $z$ where correlations between the ions are small (which justifies the mean-field approximation) but where local charge neutrality is still valid. The physical reason for the much slower decay of the colloidal density profiles in the second regime results from the counterion entropy which tends to delocalize the counterions. However, since the macroions are coupled to the counterions due to the constraint of local charge neutrality, they are lifted upwards together with their counterions. Assuming that the main contribution in the integrand of the right-hand-side of (1) comes from the second regime, the mean heights are given by $h_{\alpha}=1 / \gamma_{\alpha}$ and $h_{\beta}=1 / \gamma_{\beta}$. A brazil nut effect occurs when $\alpha=1$ and $\beta=2$. Hence the transition towards the brazil nut effect happens at $\frac{m_{1}}{Z_{1} / q+1}=\frac{m_{2}}{Z_{2} / q+1}$.

Let us now test the prediction of the theory against our simulation data. First, the slopes in the inset of Figure 1 confirm the inverse decay lengths $\gamma_{\alpha}$ and $\gamma_{\beta}$ perfectly

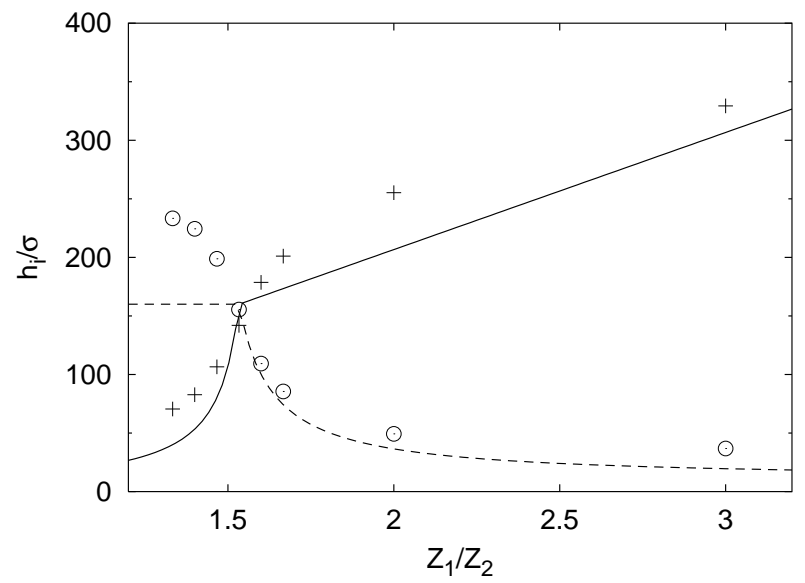

FIG. 3: Mean heights $h_{i}$ of heavy $(+)$ and light $(\odot)$ particles as a function of $Z_{1}$ for the parameters of Fig. 11 The lines show the theoretical predictions.

for a large range of intermediate heights; the theoretical predictions for the slopes as given by Eqs. (3) and (4) are shown as thick lines. The crossover to the bare gravitational length for large $z$ is confirmed for the highcharge particles and was found to be pretty sharp. Consistently with the theoretical assumption, local charge neutrality in the intermediate regime is fulfilled. Second, we have tested the location of the transition towards the colloidal brazil nut effect by systematically varying the mass and charge ratio. The results are summarized in Figure 2 The theory, which predicts the transition at $\left(Z_{1}+1\right) /\left(Z_{2}+1\right)=m_{1} / m_{2}$, is shown as a straight line there. All parameter combinations simulated are indeed separated by this theoretical prediction, confirming our simple theory. This is remarkable as any wall or bulk correlation effects are neglected in the theory.

In order to elucidate this further, we have compared the theoretical predictions $1 / \gamma_{1}, 1 / \gamma_{2}$ for the heights with the simulation data in a situation where the transition line was crossed. In Figure 3 the heights are shown as a function of a varied charge $Z_{1}$. The simulation data reveal that the variation of both heights $h_{i}$ with $Z_{1}$ is large close to the transition and becomes maximal at the transition. In the theory, this feature is reproduced and accompanied by a generic cusp at the transition. Though the cusp is smeared out and in general the heights are larger in the simulation data due to the density reduction close to the wall, there is still semi-quantitative agreement. The rapid variation of the heights at the transition implies that the location of the transition towards the brazil nut effect is very robust explaining the validity of the theory in Figure 2

Finally we address the case of added monovalent salt. Density profiles for the two macroion species and the counter- and coions are presented in Figure 4. Addition of salt reduces the brazil nut effect; in the inset of Figure 4 the heights are plotted versus added salt concentration. The threshold salt concentration $c_{s}^{(0)}$ 


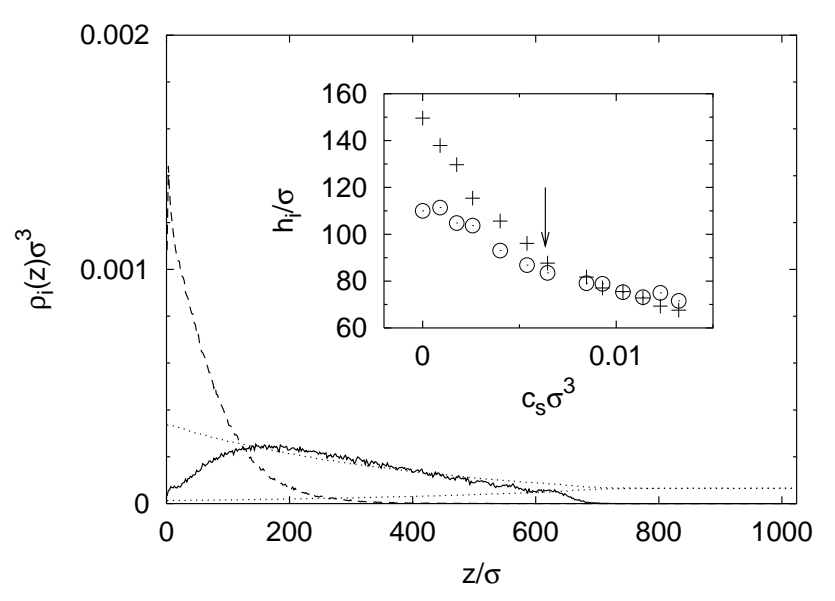

FIG. 4: Density profiles for added salt. The counter- and coion densities have been divided by $Z_{1}+Z_{2}$, the coion density is smaller than that of the counterions. The parameters are $Z_{1}=45, m_{1} / m_{2}=2, \ell_{2}=20 \sigma$, and $c_{s} \sigma^{3}=2.5 \times 10^{-3}$. Inset: Sedimentation heights $h_{i}$ of heavy $(+)$ and light $(\odot)$ particles as a function of reduced salt concentration for $Z_{1}=$ $32, m_{1} / m_{2}=2$, and $\ell_{2}=5 \sigma$. The theoretical estimate of the threshold salt concentration is marked by an arrow.

needed to reverse the brazil nut effect is estimated as $c_{s}^{(0)} \approx Z_{1} \rho_{1}\left(z_{0}\right)+Z_{2} \rho_{2}\left(z_{0}\right)$ which is the counterion concentration at the position $z_{0}$ where the two colloidal density profiles cross in the salt-free case. This salt concentration is indicated as an arrow in the inset of Figure 4 confirming the validity of the estimate. Though the brazil nut effect is proven to remain stable with respect to added salt, it will only show up for deionized solutions. Highly charged suspensions in non-polar solvents with a small dielectric constant $\epsilon$ and low impurity ion concentrations [20] are promising candidates to exhibit a strong brazil nut effect.

In conclusion, we predict an analog of the granular brazil nut effect in equilibrium sediments of charged suspensions, which is generated by an entropic charge lifting due to the Coulomb coupling to the counterions. An experimental verification of the levitation should be possible employing depolarized light scattering [9] or confocal microscopy [20]. The simulated charge asymmetries correspond to micelles and proteins rather than highly charged colloids but the simple theory which was confirmed by the simulations is applicable for arbitrary charges.

The lifting effect is immediately generalizable to sediments of solutions which are polydisperse in mass and charge 21]. Therefore the colloidal brazil nut effect has important biochemical implications for the separation of polydisperse biological matter, such as protein solutions. Analytical sedimentation is typically used in an ultracentrifuge to separate different species 22]. The colloidal brazil nut effect implies that the separation is sensitive to the mass per charge but not to the mass itself.

We are grateful to T. Palberg, F. Scheffold, A. Philipse, C.N. Likos, R. van Roij, and R. Piazza for helpful discussions. Financial support within the Deutsche Forschungsgemeinschaft (wetting priority program and SFB TR6) is gratefully acknowledged.
[1] J. C. Williams, Powder Technol. 15, 245 (1976).

[2] A. Rosato, K. J. Strandburg, F. Prinz, R. H. Swendsen, Phys. Rev. Lett. 58, 1038 (1987).

[3] T. Shinbrot, F. J. Muzzio, Phys. Rev. Lett. 81, 4365 (1998).

[4] D. C. Hong, P. V. Quinn, S. Luding, Phys. Rev. Lett. 86, 3423 (2001).

[5] J. A. Both, D. C. Hong, Phys. Rev. Lett. 88, 124301 (2002).

[6] A. P. J. Breu, H.-M. Ensner, C. A. Kruelle, I. Rehberg, Phys. Rev. Lett. 90, 014302 (2003).

[7] V. Lobaskin, A. Lyubartsev, P. Linse, Phys. Rev. E 63, 020401 (2001).

[8] M. A. Rutgers, J. H. Dunsmuir, J.-Z. Xue, W. B. Russel, P. M. Chaikin, Phys. Rev. B 53, 5043 (1996).

[9] R. Piazza, T. Bellini, V. Degiorgio, Phys. Rev. Lett. 71, 4267 (1993).

[10] A.P. Philipse and G.H. Koenderink, Adv. Colloid Interface Sci. 100, 613 (2003).

[11] A. Einstein, Annalen der Physik 17, 549 (1905); 19, 371 (1906); J. Perrin, J. Physique 9, 5 (1910).

[12] T. Biben, J.-P. Hansen, J. Phys. Condensed Matter 6, A345 (1994).

[13] J.-P. Simonin, J. Phys. Chem. 99, 1577 (1995).
[14] H. Löwen, J. Phys.: Condensed Matter 10, L479 (1998).

[15] G. Tellez, T. Biben, Europ. Phys. J. E 2, 137 (2000).

[16] R. van Roij, J. Phys.: Condens. Matter 15, S3569 (2003).

[17] J. Lekner, Physica A 176, 485 (1991).

[18] T. Biben, J. P. Hansen, Mol. Phys. 80, 853 (1993); H. Walliser, Phys. Rev. Lett. 89, 189603 (2002).

[19] The charge non-neutrality at the system boundaries will result in a small electric field as recently predicted by theory in Ref. [16] and also confirmed by simulation of the one-component colloidal system (A.-P. Hynninen, R. van Roij, M. Dijkstra, private communication). The presence of this field, however, will change neither the density field for intermediate $z$ nor the general conclusions of our analysis which assumes local charge neutrality.

[20] C. P. Royall, M. E. Leunissen, A. van Blaaderen, J. Phys.: Condensed Matter 15, S3581 (2003).

[21] L. Bellier-Castella, H. Xu, J. Phys.: Condensed Matter 15, 5417 (2003).

[22] S.E. Harding, A.J. Rowe, J.C. Norton (Eds.): Analytical Ultracentrifugation in Biochemistry and Polymer Science, Cambridge: The Royal Society of Chemistry, 1992. 